\title{
Research on Construction Technology of Optical Radiation Measuring System Based on Virtual Instrument Technology
}

\author{
Sanfeng Dong \\ xijing university \\ Xijing University school fund number xj130103 \\ hunter2011@foxmail.com
}

Keywords: Optical radiation, Measurement, Virtual instrument, Measuring system, Uncertainty.

\begin{abstract}
Using virtual instrument technology, digital signal processing technology and traditional optical radiation measuring technology to construct optical radiation measuring system breaks the construction methods of traditional instruments. Signal processing, collection, control and process of measuring system are implemented by the software LabVIEW8.2. And they are integrated in a computer. The computer not only is data processing center, but also is instrument control center. While measuring, the user uses the mouse to operate the handles including knobs, switch and buttons of virtual instrument panel to select instrument functions and set various parameters, which realizes measuring optical radiation with different wave bands and different intensity. And the user can change instrument operation panel, modify system software, transform instrument function, and customize instrument parameters, which embodies the idea that the software is the instrument.
\end{abstract}

\section{Introduction}

Different radiometric quantities of traditional optical radiation measurement have the corresponding measuring methods in different spectrum s. Using the modern technologies including virtual instrument technology and digital signal processing technology to construct optical radiation measuring system breaks the construction methods of traditional instruments. The collection and process of electric signals in the test is realized by the software, and they are integrated in a computer. The computer not only is data processing center, but also is instrument control center. While measuring, the user uses the mouse to operate the handles including knobs, switch and buttons of virtual instrument panel to select instrument functions and set various parameters, which realizes measuring optical radiation with different wave bands and different intensity. The middle parameters, final results and waveforms in the test can be analyzed, processed, displayed and printed by the computer, which makes full use of data process and display of the computer.

\section{Construction of Optical Radiation Measuring System Based on Virtual Instrument Technology}

Optical radiation measuring system based on virtual instrument technology includes virtual measurement system and actual signal measurement system. Virtual measurement system is a simulation system consisting of virtual light source, virtual monochromator, virtual chopper, virtual detector and computer-control system. Actual signal measurement system is the system that completes the same functions of traditional optical radiation measuring system, and it collects, tests and processes actual signals. The system includes actual light source, concentrator system. chopper, monochromator, detector, data acquisition card and computer-control system.

Computer-control system is the sharing system of actual system and virtual system, and is the core of the paper. The system includes signal processing, data control and systematic uncertainty. Signal processing means band-pass filter, coherence filter and lock-in amplifier which are realized by the softwares on LabVIEW platform filter, extract and process the signals. The system control means the control on chopper and grating monochromator based on LabVIEW platform. And 
systematic uncertainty means that the evaluation unit uses $\mathrm{A}$ and $\mathrm{B}$ rating method to evaluate the designed system performance automatically.

Table 1 is the parameters of common pyroelectric materials. When $\Delta T$ is smaller, $d P_{S} / d T$ can be seen as the constant. The output signal $\Delta V$ is proportional to the velocity of temperature variation and is independent of the condition if crystal and radiation reach thermal equilibrium. The voltage generated by the detector in the spectrum is independent of the wavelength, and it is non-preference detector.

Table 1 Parameters of common pyroelectric materials

\begin{tabular}{|c|c|c|c|c|c|}
\hline Materials & $\begin{array}{l}\text { Curie } \\
\text { temperatur } \\
\text { e }(\mathrm{K})\end{array}$ & $\begin{array}{l}\text { Operating } \\
\text { temperature } \\
(\mathrm{K})\end{array}$ & $\begin{array}{l}\text { Pyroelectric } \\
\text { coefficient } \\
\left(\mathrm{cm}^{-2} \mathrm{~K}^{-1}\right)\end{array}$ & $\begin{array}{l}C_{p} \\
\left(J_{C m}^{-3} K^{-1}\right)\end{array}$ & $\begin{array}{l}1 \\
\text { resistivity } \\
(\Omega \mathrm{cm})\end{array}$ \\
\hline \multirow[t]{2}{*}{$\mathrm{BaTiO}_{3}$} & 399 & 300 & $2 \times 10^{-8}$ & $\approx 2$ & $\approx 10^{12}$ \\
\hline & & 333 & $7 \times 10^{-8}$ & $\approx 2$ & $\approx 10^{12}$ \\
\hline $\mathrm{LiTaO}_{3}$ & 891 & 300 & $1.9 \times 10^{-8}$ & $\approx 3$ & $\approx 10^{12}$ \\
\hline TGS & 322 & 300 & $3 \times 10^{-8}$ & $\approx 1.8$ & $\approx 10^{12}$ \\
\hline \multirow{2}{*}{$\begin{array}{l}\mathrm{Sr}_{1-x} \mathrm{Ba}_{x} \mathrm{Nb}_{2} \mathrm{O}_{6} \\
x=0.33 / x=0.4\end{array}$} & 335 & 300 & $1.1 \times 10^{-8}$ & $\approx 2$ & $1.2 \times 10^{8}$ \\
\hline & 351 & 300 & $8.5 \times 10^{-8}$ & $\approx 2$ & $1.6 \times 10^{8}$ \\
\hline
\end{tabular}

When multi-points output, the pyroelectric coefficient $d P_{S} / d T$ and the temperature are stored in the program in advance with the change rate $d T / d t$ of the time. The sensitive area $A$ and load resistance $R_{L}$ of the detector are inputted by the user.

\section{Speed Control of Electromotor}

Dynamic characteristics of stepping motor. When electromotor runs, the motor rotor must generate large enough moment to overcome friction and acceleration mass. If the motor can't generate large enough moment, it may cause the stall of electromotor, which makes rotor stepper and phase excitation fall out and causes incorrect load position.

The starting torque-frequency characteristics indicate that in the rage of I area, the stepping motor can stop or restart, or turns back, which makes out-of-step impossible. II area is one-way working area in which if the motor doesn't stop, restart and reverse, it can't be out of step. III area is out-of-step area, in which it can't be out of step in any conditions. In order t o work in II area, the motor must work in I area firstly, then the acceleration slope is used to change to II area.

Running torque-frequency characteristics indicate that the higher the starting engine speed, the smaller the starting torque, But when the starting engine speed is higher, it is easy to be out of step. So we should take measures of speed control for stepping motor.

Acceleration control algorithm of stepping motor. For the control system of stepping motor, when an electric pulse is given, the stepping motor moves an angle-or goes ahead for one step. The input is pulse number $\mathrm{N}$, the output is the angle $\theta$, and proportional relation between the input and output of stepping motor is $\theta=\theta_{s} N$ in which $\theta_{s}$ is stepping angle.

The kinetic equation of rotating objects of stepping motor is

$$
T=J \frac{d^{2} \theta}{d t^{2}}+B \frac{d \theta}{d t}+T_{L}
$$

In the formula, $T —$ output torque

$J$ _ rotational inertia of rotor

$B$ __amping coefficient

$T_{L} \_$load torque

$\theta \_$rotor position. 
If the velocity of rotating angle, $\omega$, has been know, $\omega=\frac{d \theta}{d t}=\theta_{s} \frac{d N}{d t}$, the driving pulse frequency $\frac{d N}{d t}$ is represented by $f, \omega=\theta_{s} f$. And the above equation can be represented as follows.

$$
T=J \theta_{s} \frac{d f}{d t}+B \theta_{s} f+T_{L}
$$

If the motor runs, it must generate the largest torque under all frequencies,

$$
J \theta_{s} \frac{d f}{d t}+B \theta_{s} f+T_{L}<T_{M}
$$

In the formula, $T_{M}$ is the output torque of stepping motor.

If the torque-frequency characteristic curve is declining linearly, the output torque of the motor can be solved by the following formula.

$$
T_{M}=T_{M 0}-k f \text {, in which } T_{M 0} \text { is the largest torque, } k \text { is the }
$$
slope, $k=\left(T_{M 0}-T_{L}\right) / f_{m}$ and $f_{m}$ is the highest running frequency of load $T_{L}$ stepping motor.

In the range of working speed, the motion equation is

$$
J \theta_{s} \frac{d f}{d t}+B \theta_{s} f+T_{L}<T_{M 0}-k f
$$

If the motor raises speed from 0 , the initial value $t=0, f=0$, the solution of the equation $J \theta_{s} \frac{d f}{d t}+\left(B \theta_{s}+k\right) f-\left(T_{M 0}-T_{L}\right)=0$ is

$$
f=f_{c}\left(1-e^{-t / \tau}\right)
$$

The formula (4) indicates that the frequency $f$ of driving pluses should rise with the time $\mathrm{t}$, which makes the rotation speed of stepping motor rises to the required running speed in a short time.

In the formula, $\tau=\frac{J \theta_{s}}{B \theta_{s}+k}$ is the time constant determining the raising speed, $f_{c}=\frac{T_{M 0}-T_{L}}{B \theta_{s}+k}$.

Discrete processing in the process of stepping motor raising and reducing speed. If damping torque is ignored and the damping coefficient $B=0, f_{c}=f_{m}$, in which $f_{m}$ is the intersection point of load torque frequency characteristic and pull- out torque frequency characteristic, which means that pull- out torque reduced to that under the point which is the same to lad torque with the increase of frequency. The stepping motor must run in the condition that it is lower than the frequency, which ensures not out of step. The actual $f_{c}$ should be less than $f_{m}$.

From formula (4), we can get

$$
f_{r}=f_{m}-f_{m} e^{-t / \tau}
$$

And the time of raising speed is

$$
t_{r}=\tau\left[\ln f_{m}-\ln \left(f_{m}-f_{r}\right)\right]
$$

The approximation method of step curve is that raising speed section is divided into $n$ sections evenly. If the rise time is $t_{r}$, the time interval between two velocity changes is $\Delta t=t_{r} / n$, and the frequency of each gear is

$$
f_{i}=f_{m}-f_{m} e^{-(i \Delta t) / \tau}(i=0,1 \cdots \cdots, n)
$$

The step number $N_{i}$ of each gear operating speed is

$N_{i}=f_{i} \times \Delta t=f_{i} t_{r} / n$

The total step number of raising speed is 


$$
N_{r}=\sum_{i=1}^{n} N_{i}
$$

The less $n$ is, the more rapid the raising speed, and the more easy the out of step. The greater $n$ is, the more stationary the operation of the stepping motor, and the approximation curve 2 is closer to the ideal curve 1. But the increase of the time of raising speed affects the capability of CPU processing other events. For the actual value, $f_{i}-f_{i-1}<f_{q} \cdot f_{q}$ is the allowed sudden-jump frequency of the driving system. And it not only can be obtained by the starting torque-frequency characteristic curve of the motor and that of the load, but also can be achieved by the maximum

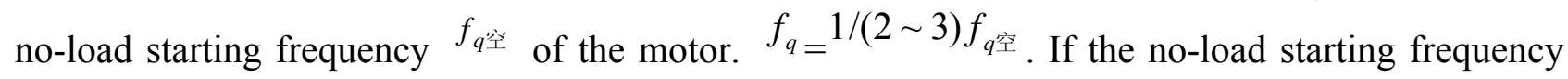
$f_{q \text { 空 }}$ of the stepping motor is $1500 \mathrm{~Hz}, f_{q}=0.5 f_{q \text { 空 }}=750 \mathrm{~Hz}$.

The processing method of reducing speed is the same to that of raising speed, which is the inverse process of raising speed. The reduction of speed starts from $f_{g}$ uniformly, and the curve of reducing speed is

$$
f_{d}=f_{g} e^{-t / \tau}
$$

In the formula, $f_{g}$ is the velocity of uniform process, and $\tau$ is generally taken the value which is the same to the process of raising speed.

Design of raising and reducing speed program of stepping motor. Calculation of operating parameters in the process of stepping motor raising and reducing speed

The operating parameters in the process of stepping motor raising and reducing speed are calculated on LabVIEW platform. The input controls include the step number, the maximum operating frequency $f_{m}$, the lowest impulse frequency of motor, the maximum non-load starting frequency of stepping motor, the raising time and the time constant. The output controls are two dimensional arrays in which one displays the raising speed, and the other shows the step number of raising speed. For the tranching number of input step, if the values the following three conditions appear for the input, the program reminds the error. (1) The frequency of the gear is less than the minimal pulse frequency of stepping motor, (2) The frequency of the gear is greater than the lowest starting frequency of stepping motor, (3) The difference of two adjacent is greater than the lowest starting frequency of stepping motor.

The technical data of stepping data with the type of $110 \mathrm{BF} 003$ is selected as the data input. It is common that the number of gears of input step is 20-1000, the maximal operation frequency $f_{m}$ is $3000-8000$, and the maximal non-load starting frequency of stepping motor is 5000 .

The program design for the process of reducing speed is similar to that of raising speed, and the functions and realization methods are similar.

Analysis on the process of raising and reducing speed

We can get from discretional step curve of raising and reducing speed several conditions in the process of raising and reducing speed, as follows. When $f_{m}<f_{0}$, there is no need to raise and reduce the speed, and it only needs to take uniform velocity to operate and complete the task. When $f_{m}>f_{0}$, the process of raising and reducing speed is considered to be used. When $N_{r}+N_{c}+N_{f}>N_{r}+N_{f}$, it needs the process of raising and reducing speed and several uniform processes. When $N_{r}+N_{c}+N_{f} \leq N_{r}+N_{f}$, the stepping motor reduces the speed without accelerating to $f_{m}$, and doesn't need the uniform process.

Program design of the process of raising and reducing speed

Program design of the process of raising and reducing speed is completed by taking three sequential structures. Firstly, the arrays and waveforms generated by the ascent algorithm are used. Then, the last value of the array is taken as the speed value of the uniform velocity, and the step 
number of the process is the same to that in the process of raising speed. Lastly, the speed is reduced according to the declining algorithm.

\section{Realization of Dual Phase Amplifier Based on Virtual Instrument Technology}

Dual phase amplifier based on virtual instrument technology is base4d on basic phase-locked amplifier, which increases the composition of band-pass filter. So the basic phase-locked amplifier based pm LabVIEW is the core module of lock-in amplifier consisting of phase locked loop / wave shaping, two phase shifters, two correlators /synchronous integrator, two phase-sensitive detectors and amplitude phase difference calculation.

The basic structure of the module is similar to that of common dual phase lock-in amplifier. It includes the most basic parts of dual phase lock-in amplifier, phase shifter, correlator, amplitude and phase calculation. Each module is one sub- VI which adjusts the submodules to complete the task of dual phase lock-in amplifier.

\section{System Integration}

Optical radiation measurement system consists of three modules, as shown in Figure 1, signal input module, test function and parameter selection module, and test result output and assessment module.

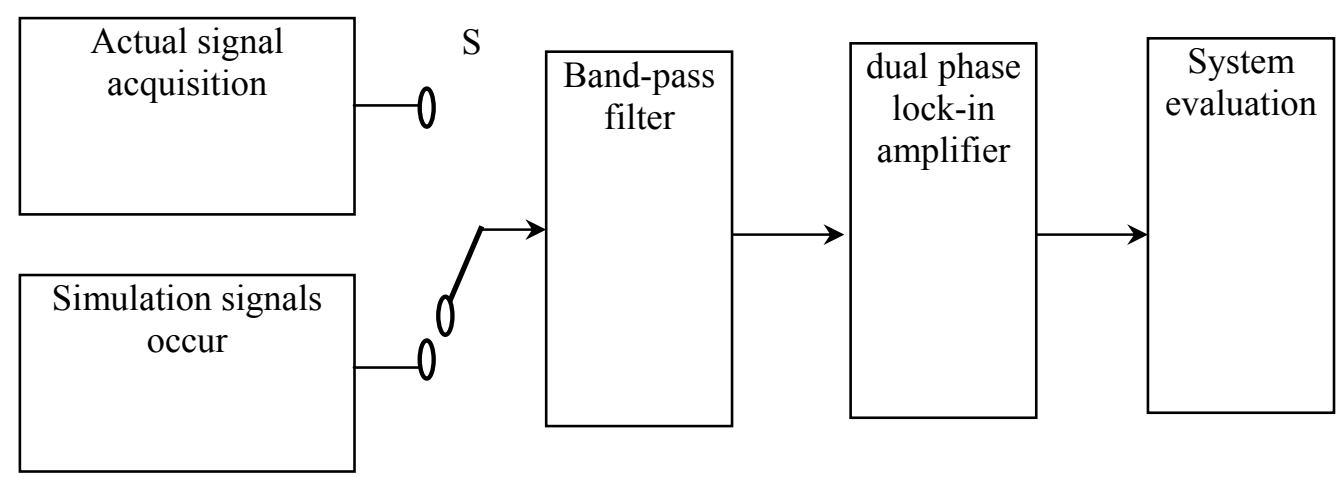

Fig. 1 Composition of optical radiation measurement system

Simulation Signals and Reference Signals. The main input controls of simulation signals and reference signals include

(1) The effective value of the measured signals. It is used to input the effective value of the measured signals.

(2) Input SNR. The ratio between the effective value of the measured simulation signals and that of noise signals determines the standard deviation of gaussian noise signals.

(3) Signal type. The reference signals and simulation signals are selected. And sinusoidal wave and square wave are selected.

(4) Sampling information.It includes sampling number and the number of one-frame waveform samples, which samples the information of the measured simulation signals.

(5) Signal frequency. The frequency of the measured simulation signals is input.

(6) Frequency jitter. The percent values of frequency jitter of the measured simulation signals are input.

(7) Effective values of reference signals. It is used to input the effective values of the reference signals.

(8) Fshakphin. It means the initial phase represented by the angle.

And the main output controls and functions are as follows.

(1) Three wave displays. They display phase change and the output waveform of composite signals including reference signals and noise signals.

(2) Fshakphout. It means phase output signals. 
(3) The real-time phase of signal output.

Actual test signal collection. The frequency of the collected sinusoidal signal in the front panel of the actual test signal collection is $15 \mathrm{~Hz}$, and the amplitude of input signals is $100 \mathrm{mv}$.

The main input controls include

(1) Sample mode selection. Continuous sampling and single point sampling can be used.

(2) Input mode selection.

(3) Sampling number. It means the number of the collected samples.

(4) Sampling rate. It is used to input the rate of system acquisition.

(5) The actual input channel. The names of channels used for input are selected.

(6) The number of channels. It means the number of channels used for input in data acquisition card.

(7) Setting of input maximum and minimum. The amplitude of the actual input signals is limited.

\section{Conclusion}

Using virtual instrument technology, digital signal processing technology and traditional optical radiation measuring technology to construct optical radiation measuring system not only breaks the construction methods of traditional instruments, but also embodies the thought that the software is the instrument. And the computer not only is data processing center, but also is instrument control center. The construction method of the optical radiation measurement system based on virtual instrument technology not only expands the range of measuring optical radiation, but also improves the reliability, accuracy and the test automation degree. According to test requirements, the user c an change instrument operation panel, modify system software and change instrument functions, which embodies the idea that the software is the instrument. Based on analysis on the technology of traditional optical radiation test, the modern electronic measurement technique, modern digital processing technique and optical test technology are combined, and the digital processing and display function of the computer is fully used, which not only is a new mode of optical radiation measurement technology, but also is one of the development directions of optical radiation measuring technology.

\section{Reference}

[1] Q.R. Lu, Y.J. Xu, S.N. Li, J. Wei, Intelligent control of liquid level of water electrical appliances, Chinese Journal of Scientific Instrument 2007 (4):788-790

[2] Q.R. Lu, S.L. Zhuang, Realization of evaluation methods on systematic uncertainty based on LabVIEW platform, Journal of Guilin Institute of Technology, 2007, No.4:584-588.

[3] Q.R. Lu, S.L. Zhuang, Research on solution of survivability problems of optical fiber transmission network, Optical Communication Technique, 2007, 9:29-31.

[4] Q.R. Lu, Observation on construction methods of economic FTTH system, Optical Communication Technique, 2006, 7:22-24.

[5] Q.R. Lu, Z.M. Zou, Design and realization of logic analyzer based on dual CPU, Chinese Journal of Scientific Instrument, 2006 (6) , 1523-1524+1540.

[6] Q.R. Lu, Design and realization of economic logic analyzer, Journal of Guilin Institute of Technology, 2006, No.3:415-418

[7] Q.R. Lu, Construction of personal laboratory based on virtual instrument technology, Beijing, Electronic Industry Press, 2006.10. 